\title{
Some properties of the generalized Fibonacci and Lucas sequences related to the extended Hecke groups
}

\author{
Sebahattin Ikikardes $^{1 *}$ and Zehra Sarıgedik² \\ Dedicated to Professor Hari M Srivastava
}

\author{
${ }^{*}$ Correspondence: \\ skardes@balikesir.edu.tr \\ ${ }^{1}$ Fen-Edebiyat Fakültesi, Matematik \\ Bölümü, Balıkesir Üniversitesi, \\ Balıkesir, 10145, Turkey \\ Full list of author information is \\ available at the end of the article
}

\begin{abstract}
In this paper, we define a sequence, which is a generalized version of the Lucas sequence, similar to the generalized Fibonacci sequence given in Koruoğlu and Şahin in Turk. J. Math. 2009, doi:10.3906/mat-0902-33. Also, we give some connections between the generalized Fibonacci sequence and the generalized Lucas sequence, and we find polynomial representations of the generalized Fibonacci and the generalized Lucas sequences, related to the extended Hecke groups given in Koruoğlu and Şahin in Turk. J. Math. 2009, doi:10.3906/mat-0902-33.

MSC: $20 \mathrm{H} 10 ; 11 \mathrm{FO}$
\end{abstract}

Keywords: extended Hecke group; generalized Fibonacci sequence; generalized Lucas sequence

\section{Introduction}

In [1], Hecke introduced groups $H(\lambda)$, generated by two linear fractional transformations

$$
T(z)=-\frac{1}{z} \quad \text { and } \quad S(z)=-\frac{1}{z+\lambda},
$$

where $\lambda$ is a fixed positive real number. Hecke showed that $H(\lambda)$ is discrete if and only if $\lambda=\lambda_{q}=2 \cos \frac{\pi}{q}, q \in \mathbb{N}, q \geq 3$, or $\lambda \geq 2$. These groups have come to be known as the Hecke Groups, and we will denote them $H\left(\lambda_{q}\right), H(\lambda)$ for $q \geq 3, \lambda \geq 2$, respectively. The Hecke group $H\left(\lambda_{q}\right)$ is the Fuchsian group of the first kind when $\lambda=\lambda_{q}$ or $\lambda=2$, and $H(\lambda)$ is the Fuchsian group of the second kind when $\lambda>2$. In this study, we focus on the case $\lambda=\lambda_{q}$, $q \geq 3$. The Hecke group $H\left(\lambda_{q}\right)$ is isomorphic to the free product of two finite cyclic groups of orders 2 and $q$, and it has a presentation

$$
H\left(\lambda_{q}\right)=\left\langle T, S \mid T^{2}=S^{q}=I\right\rangle \cong C_{2} * C_{q}, \quad[2] .
$$

The first several of these groups are $H\left(\lambda_{3}\right)=\Gamma=\operatorname{PSL}(2, \mathbb{Z})$ (the modular group), $H\left(\lambda_{4}\right)=$ $H(\sqrt{2}), H\left(\lambda_{5}\right)=H\left(\frac{1+\sqrt{5}}{2}\right)$, and $H\left(\lambda_{6}\right)=H(\sqrt{3})$. It is clear that $H\left(\lambda_{q}\right) \subset \operatorname{PSL}\left(2, \mathbb{Z}\left[\lambda_{q}\right]\right)$, for $q \geq 4$. The groups $H(\sqrt{2})$ and $H(\sqrt{3})$ are of particular interest, since they are the only Hecke groups, aside from the modular group, whose elements are completely known (see, [3]).

\section{Springer}

C 2013 Ikikardes and Sarıgedik; licensee Springer. This is an Open Access article distributed under the terms of the Creative Commons Attribution License (http://creativecommons.org/licenses/by/2.0), which permits unrestricted use, distribution, and reproduction in any medium, provided the original work is properly cited. 
The extended Hecke group, denoted by $\bar{H}\left(\lambda_{q}\right)$, has been defined in [4] and [5] by adding the reflection $R(z)=1 / \bar{z}$ to the generators of the Hecke group $H\left(\lambda_{q}\right)$. The extended Hecke group $\bar{H}\left(\lambda_{q}\right)$ has a presentation

$$
\left\langle T, S, R \mid T^{2}=S^{q}=R^{2}=I, R T=T R, R S=S^{q-1} R\right\rangle \cong D_{2} *_{\mathbb{Z}_{2}} D_{q} .
$$

The Hecke group $H\left(\lambda_{q}\right)$ is a subgroup of index 2 in $\bar{H}\left(\lambda_{q}\right)$. It is clear that $\bar{H}\left(\lambda_{q}\right) \subset$ $\operatorname{PGL}\left(2, \mathbb{Z}\left[\lambda_{q}\right]\right)$ when $q>3$ and $\bar{H}\left(\lambda_{3}\right)=\operatorname{PGL}(2, \mathbb{Z})$ (the extended modular group $\left.\bar{\Gamma}\right)$.

Throughout this paper, we identify each matrix $A$ in $\operatorname{GL}\left(2, \mathbb{Z}\left[\lambda_{q}\right]\right)$ with $-A$, so that they each represent the same element of $\bar{H}\left(\lambda_{q}\right)$. Thus, we can represent the generators of the extended Hecke group $\bar{H}\left(\lambda_{q}\right)$ as

$$
T=\left(\begin{array}{cc}
0 & -1 \\
1 & 0
\end{array}\right), \quad S=\left(\begin{array}{cc}
0 & -1 \\
1 & \lambda_{q}
\end{array}\right) \quad \text { and } \quad R=\left(\begin{array}{ll}
0 & 1 \\
1 & 0
\end{array}\right) .
$$

In [6], Koruoglu and Sahin found that there is a relationship between the generalized Fibonacci numbers and the entries of matrices representations of some elements of the extended Hecke group $\bar{H}\left(\lambda_{q}\right)$. For the elements

$$
h=T S R=\left(\begin{array}{cc}
\lambda_{q} & 1 \\
1 & 0
\end{array}\right) \quad \text { and } f=R T S=\left(\begin{array}{cc}
0 & 1 \\
1 & \lambda_{q}
\end{array}\right)
$$

in $\bar{H}\left(\lambda_{q}\right)$, then the $k$ th power of $h$ and $f$ are

$$
h^{k}=\left(\begin{array}{cc}
a_{k} & a_{k-1} \\
a_{k-1} & a_{k-2}
\end{array}\right) \quad \text { and } \quad f^{k}=\left(\begin{array}{cc}
a_{k-1} & a_{k} \\
a_{k} & a_{k+1}
\end{array}\right)
$$

where $a_{0}=0, a_{1}=1$, and for $k \geq 2$,

$$
a_{k}=\lambda_{q} a_{k-1}+a_{k-2}
$$

For all $k \geq 2$,

$$
a_{k}=\frac{1}{\sqrt{\lambda_{q}^{2}+4}}\left[\left(\frac{\lambda_{q}+\sqrt{\lambda_{q}^{2}+4}}{2}\right)^{k+1}-\left(\frac{\lambda_{q}-\sqrt{\lambda_{q}^{2}+4}}{2}\right)^{k+1}\right] .
$$

Notice that this real numbers sequence is a generalized version of the common Fibonacci sequence. If $\lambda_{q}=1$, this sequence coincides with the Fibonacci sequence.

The Fibonacci and the Lucas sequence have been studied extensively and generalized in many ways. For example, you can see in [7-12]. In this paper, firstly, we define a sequence $b_{k}$, which is a generalization of the Lucas sequence. Then we give some properties of these sequences and the relationships between them. To do this, we use some results given in [13-15]. In fact, in [14] and [15], Özgür found two sequences, which are the generalization of the Fibonacci sequence and the Lucas sequence, in the Hecke groups $H(\lambda), \lambda \geq 2$ real. But the Hecke groups $H(\lambda)$ are different from the Hecke groups $H\left(\lambda_{q}\right), \lambda_{q}=2 \cos \frac{\pi}{q}, q \in \mathbb{N}$, $q \geq 3$. 


\section{Some properties of generalized Fibonacci and generalized Lucas sequences}

Firstly, we define a sequence $b_{k}$ by

$$
b_{k}=\lambda_{q} b_{k-1}+b_{k-2}
$$

for $k \geq 2$, where $b_{0}=2, b_{1}=\lambda_{q}$.

Proposition 1 For all $k \geq 2$,

$$
b_{k}=\left(\frac{\lambda_{q}+\sqrt{\lambda_{q}^{2}+4}}{2}\right)^{k}+\left(\frac{\lambda_{q}-\sqrt{\lambda_{q}^{2}+4}}{2}\right)^{k} .
$$

Proof To solve (6), let $b_{k}$ be a characteristic polynomial $r^{k}$. Then we have the equation

$$
r^{k}=\lambda_{q} r^{k-1}+r^{k-2} \Rightarrow r^{2}-\lambda_{q} r-1=0
$$

The roots of this equation are

$$
r_{1,2}=\frac{\lambda_{q} \pm \sqrt{\lambda_{q}^{2}+4}}{2}
$$

Using these roots $r_{1,2}$, we can find a general formula of the general term $b_{k}$. If we write $b_{k}$ as combinations of the roots $r_{1,2}$, then we have

$$
b_{k}=A\left(\frac{\lambda_{q}+\sqrt{\lambda_{q}^{2}+4}}{2}\right)^{k}+B\left(\frac{\lambda_{q}-\sqrt{\lambda_{q}^{2}+4}}{2}\right)^{k}
$$

To determine constants $A$ and $B$, we use two boundary conditions $b_{0}=2$ and $b_{1}=\lambda_{q}$, thus,

$$
\begin{aligned}
& b_{0}=2=A+B \\
& b_{1}=\lambda_{q}=A\left(\frac{\lambda_{q}+\sqrt{\lambda_{q}^{2}+4}}{2}\right)+B\left(\frac{\lambda_{q}-\sqrt{\lambda_{q}^{2}+4}}{2}\right) .
\end{aligned}
$$

So,

$$
\begin{aligned}
& 2 \lambda_{q}=A\left(\lambda_{q}+\sqrt{\lambda_{q}^{2}+4}\right)+(2-A)\left(\lambda_{q}-\sqrt{\lambda_{q}^{2}+4}\right) \\
& A=1 \text { and } B=1 .
\end{aligned}
$$

Then we obtain the formula of $b_{k}$ as

$$
b_{k}=\left(\frac{\lambda_{q}+\sqrt{\lambda_{q}^{2}+4}}{2}\right)^{k}+\left(\frac{\lambda_{q}-\sqrt{\lambda_{q}^{2}+4}}{2}\right)^{k} .
$$

This completes the proof. 
Notice that this formula is a generalized Lucas sequence. If $\lambda_{q}=1$ (the modular group case $q=3$ ), we get the Lucas sequence.

Now, we have two sequences $a_{k}$ and $b_{k}$, which are generalizations of the Fibonacci and the Lucas sequences. Let us write out the first 8 terms of $a_{k}$ and $b_{k}$.

$$
\begin{array}{lll}
a_{k} & b_{k} \\
a_{0}=0 & b_{0}=2 \\
a_{1}=1 & b_{1}=\lambda_{q} \\
a_{2}=\lambda_{q} & b_{2}=\lambda_{q}^{2}+2 \\
a_{3}=\lambda_{q}^{2}+1 & b_{3}=\lambda_{q}^{3}+3 \lambda_{q} \\
a_{4}=\lambda_{q}^{3}+2 \lambda_{q} & b_{4}=\lambda_{q}^{4}+4 \lambda_{q}^{2}+2 \\
a_{5}=\lambda_{q}^{4}+3 \lambda_{q}^{2}+1 & b_{5}=\lambda_{q}^{5}+5 \lambda_{q}^{3}+5 \lambda_{q} \\
a_{6}=\lambda_{q}^{5}+4 \lambda_{q}^{3}+3 \lambda_{q} & b_{6}=\lambda_{q}^{6}+6 \lambda_{q}^{4}+9 \lambda_{q}^{2}+2 \\
a_{7}=\lambda_{q}^{6}+5 \lambda_{q}^{4}+6 \lambda_{q}^{2}+1 & b_{7}=\lambda_{q}^{7}+7 \lambda_{q}^{5}+14 \lambda_{q}^{3}+7 \lambda_{q} \\
a_{8}=\lambda_{q}^{7}+6 \lambda_{q}^{5}+10 \lambda_{q}^{3}+4 \lambda_{q} & b_{8}=\lambda_{q}^{8}+8 \lambda_{q}^{6}+20 \lambda_{q}^{4}+16 \lambda_{q}^{2}+2 .
\end{array}
$$

Here, it is possible to extend $a_{k}$ and $b_{k}$ backward with the negative subscripts. For example, $a_{-1}=1, a_{-2}=-\lambda_{q}, a_{-3}=\lambda_{q}^{2}+1$, and so on. Therefore, we can deduce that

$$
a_{-k}=(-1)^{k+1} a_{k}
$$

and

$$
b_{-k}=(-1)^{k} b_{k}
$$

The sequences $a_{k}$ and $b_{k}$ have some similar properties of the Fibonacci and the Lucas numbers $F_{n}$ and $L_{n}$. Now, we investigate some properties of these sequences $a_{k}$ and $b_{k}$.

\section{Proposition 2}

$$
a_{k}+a_{k+4}=\left(\lambda_{q}^{2}+2\right) a_{k+2} \quad \text { and } \quad b_{k}+b_{k+4}=\left(\lambda_{q}^{2}+2\right) b_{k+2} .
$$

Proof We will use induction on $k$. For $k=0$, we have

$$
a_{0}+a_{4}=0+\lambda_{q}^{3}+2 \lambda_{q}=\lambda_{q}\left(\lambda_{q}^{2}+2\right)=a_{2}\left(\lambda_{q}^{2}+2\right) .
$$

For $k=1$, we get

$$
\begin{aligned}
a_{1}+a_{5} & =1+\lambda_{q}^{4}+3 \lambda_{q}^{2}+1 \\
& =\left(\lambda_{q}^{2}+2\right)\left(\lambda_{q}^{2}+1\right)=\left(\lambda_{q}^{2}+2\right) a_{3} .
\end{aligned}
$$

Now let us assume that the proposition holds for $k=2, \ldots, n$. We show that it holds for $k=n+1$. By assumption, we have

$$
a_{n-1}+a_{n+3}=\left(\lambda_{q}^{2}+2\right) a_{n+1} \text { and } a_{n}+a_{n+4}=\left(\lambda_{q}^{2}+2\right) a_{n+2} .
$$


From (3), we obtain

$$
\begin{aligned}
a_{n+1}+a_{n+5} & =\left(\lambda_{q} a_{n}+a_{n-1}\right)+\left(\lambda_{q} a_{n+4}+a_{n+3}\right) \\
& =\lambda_{q}\left(a_{n}+a_{n+4}\right)+a_{n-1}+a_{n+3} \\
& =\lambda_{q}\left(\lambda_{q}^{2}+2\right) a_{n+2}+\left(\lambda_{q}^{2}+2\right) a_{n+1} \\
& =\left(\lambda_{q}^{2}+2\right)\left(\lambda_{q} a_{n+2}+a_{n+1}\right) \\
& =\left(\lambda_{q}^{2}+2\right) a_{n+3} .
\end{aligned}
$$

Then we get

$$
a_{k}+a_{k+4}=\left(\lambda_{q}^{2}+2\right) a_{k+2}
$$

Similarly, it can be shown that

$$
b_{k}+b_{k+4}=\left(\lambda_{q}^{2}+2\right) b_{k+2}
$$

\section{Proposition 3}

$$
b_{k}=a_{k+1}+a_{k-1} \text {. }
$$

Proof We will use the induction method on $k$. If $k=1$, then

$$
b_{1}=a_{2}+a_{0} .
$$

We suppose that the equation holds for $k=2,3, \ldots, n-1$, i.e.,

$$
b_{n-1}=a_{n+1}+a_{n-1} \text {. }
$$

Now, we show that the equation holds for $k=n$. Then we have

$$
\begin{aligned}
b_{n} & =\left(\lambda_{q}^{2}+2\right) b_{n-2}-b_{n-4} \\
& =\left(\lambda_{q}^{2}+2\right)\left(a_{n-1}+a_{n-3}\right)-\left(a_{n-5}+a_{n-3}\right) \\
& =\left(\lambda_{q}^{2}+2\right) a_{n-1}-a_{n-3}+\left(\lambda_{q}^{2}+2\right) a_{n-3}-a_{n-5} \\
& =a_{n+1}+a_{n-1} .
\end{aligned}
$$

\section{Proposition 4}

$$
b_{k}+b_{k+2}=\left(\lambda_{q}^{2}+4\right) a_{k+1}
$$

Proof For $k=0$, we have

$$
\begin{aligned}
b_{0}+b_{2} & =2+\lambda_{q}^{2}+2 \\
& =\lambda_{q}^{2}+4 \\
& =\left(\lambda_{q}^{2}+4\right) a_{1} .
\end{aligned}
$$


For $k=1$, we have

$$
\begin{aligned}
b_{1}+b_{3} & =\lambda_{q}+\lambda_{q}^{3}+3 \lambda_{q} \\
& =\lambda_{q}^{3}+4 \lambda_{q} \\
& =\lambda_{q}\left(\lambda_{q}^{2}+4\right) .
\end{aligned}
$$

Now, we assume that the proposition holds for $k=2, \ldots, n$. We show that it holds for $k=$ $n+1$. By assumption, we have

$$
b_{n}+b_{n+2}=\left(\lambda_{q}^{2}+4\right) a_{n+1} \quad \text { and } \quad b_{n-1}+b_{n+1}=\left(\lambda_{q}^{2}+4\right) a_{n} .
$$

Then we find

$$
\begin{aligned}
b_{n+1}+b_{n+3} & =\left(\lambda_{q} b_{n}+b_{n-1}\right)+\left(\lambda_{q} b_{n+2}+b_{n+1}\right) \\
& =\lambda_{q}\left(b_{n}+b_{n+2}\right)+\left(b_{n-1}+b_{n+1}\right) \\
& =\lambda_{q}\left(\lambda_{q}^{2}+4\right) a_{n+1}+\left(\lambda_{q}^{2}+4\right) a_{n} \\
& =\left(\lambda_{q}^{2}+4\right)\left(\lambda_{q} a_{n+1}+a_{n}\right) \\
& =\left(\lambda_{q}^{2}+4\right) a_{n+2} .
\end{aligned}
$$

\section{Proposition 5}

$$
a_{k-3}+a_{k+3}=\left(\lambda_{q}^{2}+1\right) b_{k}
$$

Proof We will use induction on $k$. For $k=0$, we find

$$
\begin{aligned}
a_{-3}+a_{3} & =(-1)^{4} a_{3}+a_{3} \\
& =2 a_{3} \\
& =2\left(\lambda_{q}^{2}+1\right) b_{0} .
\end{aligned}
$$

For $k=1$, we get

$$
\begin{aligned}
a_{-2}+a_{4} & =(-1)^{3} a_{2}+a_{4} \\
& =-a_{2}+a_{4} \\
& =-\lambda_{q}+\lambda_{q}^{3}+2 \lambda_{q} \\
& =\lambda_{q}^{3}+\lambda_{q} \\
& =\lambda_{q}\left(\lambda_{q}^{2}+1\right) \\
& =b_{1}\left(\lambda_{q}^{2}+1\right) .
\end{aligned}
$$

Now, let us suppose that the proposition holds for $k=2, \ldots, n$. We show that it holds for $k=n+1$. By assumption, $a_{n-3}+a_{n+3}=\left(\lambda_{q}^{2}+1\right) b_{n}$ and $a_{n-4}+a_{n+2}=\left(\lambda_{q}^{2}+1\right) b_{n-1}$. Hence we 
get

$$
\begin{aligned}
a_{n-2}+a_{n+4} & =\lambda_{q} a_{n-3}+a_{n-4}+\lambda_{q} a_{n+3}+a_{n+2} \\
& =\lambda_{q}\left(a_{n-3}+a_{n+3}\right)+a_{n-4}+a_{n+2} \\
& =\lambda_{q}\left(\lambda_{q}^{2}+1\right) b_{n}+\left(\lambda_{q}^{2}+1\right) b_{n-1} \\
& =\left(\lambda_{q}^{2}+1\right)\left(\lambda_{q} b_{n}+b_{k-1}\right) \\
& =\left(\lambda_{q}^{2}+1\right) b_{n+1} .
\end{aligned}
$$

\section{Proposition 6}

$$
a_{2 k}=a_{k} b_{k} \text {. }
$$

Proof We will use the induction method on $k$. For $k=0$, we have

$$
a_{0} b_{0}=0=a_{0} .
$$

For $k=1$, we have

$$
a_{1} b_{1}=\lambda_{q}=a_{2} .
$$

We suppose that the equation holds for $k=2, \ldots, n-1$, i.e.,

$$
a_{2(n-1)}=a_{n-1} b_{n-1} .
$$

Now, we show that the equation holds for $k=n$. By equalities (3), (9) and (10),

$$
\begin{aligned}
a_{n} b_{n} & =a_{n}\left(a_{n+1}+a_{n-1}\right) \\
& =a_{n}\left(\left(\lambda_{q}^{2}+2\right) a_{n-1}-a_{n-3}\right)+a_{n-1}\left(\left(\lambda_{q}^{2}+2\right) a_{n-2}-a_{n-4}\right) \\
& =\left(\lambda_{q}^{2}+2\right) a_{n} a_{n-1}+\left(\lambda_{q}^{2}+2\right) a_{n-1} a_{n-2}-a_{n} a_{n-3}-a_{n-1} a_{n-4} \\
& =\left(\lambda_{q}^{2}+2\right) a_{n-1}\left(a_{n}+a_{n-2}\right)-a_{n} a_{n-3}-a_{n-1} a_{n-4} \\
& =\left(\lambda_{q}^{2}+2\right) a_{n-1} b_{n-1}-a_{n} a_{n-3}-a_{n-1} a_{n-4} \\
& =\left(\lambda_{q}^{2}+2\right) a_{n-1} b_{n-1}-a_{n-3}\left(\lambda_{q} a_{n-1}+a_{n-2}\right)-a_{n-1}\left(a_{n-2}-\lambda_{q} a_{n-3}\right) \\
& =\left(\lambda_{q}^{2}+2\right) a_{n-1} b_{n-1}-a_{n-3} a_{n-2}-a_{n-1} a_{n-2} \\
& =\left(\lambda_{q}^{2}+2\right) a_{n-1} b_{n-1}-a_{n-2}\left(a_{n-3}+a_{n-1}\right) \\
& =\left(\lambda_{q}^{2}+2\right) a_{n-1} b_{n-1}-a_{n-2} b_{n-2} \\
& =\left(\lambda_{q}^{2}+2\right) a_{2 n-2}-a_{2 n-4} \quad \text { (by assumption) } \\
& =a_{2 n} .
\end{aligned}
$$

\section{Proposition 7}

$$
b_{k}^{2}-\left(\lambda_{q}^{2}+4\right) a_{k}^{2}=4(-1)^{k} .
$$


Proof Using (10) and the definitions of $a_{k}$ and $b_{k}$, we have

$$
\begin{aligned}
b_{k}^{2}-\left(\lambda_{q}^{2}+4\right) a_{k}^{2} & =\left(a_{k-1}+a_{k+1}\right)^{2}-\left(\lambda_{q}^{2}+4\right) a_{k}^{2} \\
& =a_{k-1}^{2}+2 a_{k-1} a_{k+1}+a_{k+1}^{2}-\lambda_{q}^{2} a_{k}^{2}-4 a_{k}^{2} \\
& =a_{k-1}^{2}+2 a_{k-1}\left(\lambda_{q} a_{k}+a_{k-1}\right)+\left(\lambda_{q} a_{k}+a_{k-1}\right)^{2}-\lambda_{q}^{2} a_{k}^{2}-4 a_{k}^{2} \\
& =a_{k-1}^{2}+2 \lambda_{q} a_{k-1} a_{k}+2 a_{k-1}^{2}+\lambda_{q}^{2} a_{k}^{2}+2 \lambda_{q} a_{k} a_{k-1}+a_{k-1}^{2}-\lambda_{q}^{2} a_{k}^{2}-4 a_{k}^{2} \\
& =4 a_{k-1}^{2}+4 \lambda_{q} a_{k-1} a_{k}-4 a_{k}^{2} \\
& =4 a_{k-1}\left(a_{k-1}+\lambda_{q} a_{k}\right)-4 a_{k}^{2} \\
& =4 a_{k-1} a_{k+1}-4 a_{k}^{2} \\
& =4\left(a_{k-1} a_{k+1}-a_{k}^{2}\right) .
\end{aligned}
$$

In [10], Yayenie and Edson obtained a generalization of Cassini's identity for the positive real numbers $a$ and $b$. If we take $a=\lambda_{q}$ and $b=\lambda_{q}$ in generalized Cassini's identity, we get

$$
a_{k-1} a_{k+1}-a_{k}^{2}=(-1)^{n}
$$

and so,

$$
b_{k}^{2}-\left(\lambda_{q}^{2}+4\right) a_{k}^{2}=4 \cdot(-1)^{n} .
$$

\section{Proposition 8}

$$
a_{k} \cdot a_{k+3}-a_{k+1} \cdot a_{k+2}=(-1)^{k+1} \lambda_{q}
$$

Proof We will use the induction method on $k$. For $k=0$, we have

$$
a_{0} \cdot a_{3}-a_{1} \cdot a_{2}=-\lambda_{q}=(-1)^{1} \lambda_{q}
$$

For $k=1$, we have

$$
\begin{aligned}
a_{1} \cdot a_{4}-a_{2} \cdot a_{3} & =\lambda_{q}^{3}+2 \lambda_{q}-\lambda_{q}\left(\lambda_{q}^{2}+1\right) \\
& =(-1)^{2} \lambda_{q} .
\end{aligned}
$$

Now, we assume that the proposition holds for $k=2, \ldots, n$. We show that it holds for $k=$ $n+1$. From assumption $a_{n} \cdot a_{n+3}-a_{n+1} \cdot a_{n+2}=(-1)^{n+1} \lambda_{q}$, and, thus,

$$
\begin{aligned}
a_{n+1} \cdot a_{n+4}-a_{n+2} \cdot a_{n+3} & =a_{n+1}\left(\lambda_{q} a_{n+3}+a_{n+2}\right)-a_{n+3}\left(\lambda_{q} a_{n+1}+a_{n}\right) \\
& =\lambda_{q} a_{n+1} a_{n+3}+a_{n+1} a_{n+2}-\lambda_{q} a_{n+3} a_{n+1}-a_{n+3} a_{n} \\
& =a_{n+1} a_{n+2}-a_{n+3} a_{n} \\
& =-(-1)^{n+1} \lambda_{q} \\
& =(-1)^{n+2} \lambda_{q} .
\end{aligned}
$$




\section{Proposition 9}

$$
a_{2 m+2} \cdot a_{k}-a_{2 m} \cdot a_{k-2}=a_{2 m+k} \lambda_{q} .
$$

Let $m$ be fixed. We will use the induction method on $k$. For $k=0$, we have

$$
a_{2 m+2} \cdot a_{0}-a_{2 m} \cdot a_{-2}=\lambda_{q} a_{2 m}
$$

since $a_{0}=0$ and $a_{-2}=(-1)^{3} a_{2}=-\lambda_{q}$. For $k=1$, we find

$$
\begin{aligned}
a_{2 m+2} \cdot a_{1}-a_{2 m} \cdot a_{-1} & =a_{2 m+2}-a_{2 m} \\
& =\lambda_{q} a_{2 m+1}+a_{2 m}-a_{2 m} \\
& =\lambda_{q} a_{2 m+1},
\end{aligned}
$$

since $a_{1}=1$ and $a_{-1}=1$. Now, we assume that the proposition holds for $k=2, \ldots, n$. We show that it holds for $k=n+1$. By assumption,

$$
a_{2 m+2} \cdot a_{n}-a_{2 m} \cdot a_{n-2}=\lambda_{q} a_{2 m+n}
$$

and

$$
a_{2 m+2} \cdot a_{n-1}-a_{2 m} \cdot a_{n-3}=\lambda_{q} a_{2 m+n-1}
$$

Thus, we have

$$
\begin{aligned}
a_{2 m+2} \cdot a_{n+1}-a_{2 m} \cdot a_{n-1} & =a_{2 m+2}\left(\lambda_{q} a_{n}+a_{n-1}\right)-a_{2 m}\left(\lambda_{q} a_{n-2}+a_{n-3}\right) \\
& =\lambda_{q}\left(a_{2 m+2} a_{n}-a_{2 m} a_{n-2}\right)+\left(a_{2 m+2} a_{n-1}-a_{2 m} a_{n-3}\right) \\
& =\lambda_{q} \lambda_{q} a_{2 m+n}+\lambda_{q} a_{2 m+n-1} \\
& =\lambda_{q}\left(\lambda_{q} a_{2 m+n}+a_{2 m+n-1}\right) \\
& =\lambda_{q} a_{2 m+n+1} .
\end{aligned}
$$

Now, we give a formula for $a_{k}$ and $b_{k}$.

Proposition 10 For all $k \geq 1$,

$$
a_{k}= \begin{cases}\frac{1}{2^{k-1}} \sum_{i=0}^{\frac{k-2}{2}}\left(\begin{array}{c}
k \\
2 i+1
\end{array}\right) \lambda_{q}^{k-(2 i+1)}\left(\lambda_{q}^{2}+4\right)^{i} & \text { if } k \text { is even, } \\
\frac{1}{2^{k-1}} \sum_{i=0}^{\frac{k-1}{2}}\left(\begin{array}{c}
k \\
2 i+1
\end{array}\right) \lambda_{q}^{k-(2 i+1)}\left(\lambda_{q}^{2}+4\right)^{i} & \text { if } k \text { is odd }\end{cases}
$$

and

$$
b_{k}= \begin{cases}\frac{1}{2^{k-1}} \sum_{i=0}^{\frac{k}{2}}\left(\begin{array}{c}
k \\
2 i
\end{array}\right) \lambda_{q}^{k-2 i}\left(\lambda_{q}^{2}+4\right)^{2 i} & \text { if } k \text { is even }, \\
\frac{1}{2^{k-1}} \sum_{i=0}^{\frac{k-1}{2}}\left(\begin{array}{c}
k \\
2 i-1
\end{array}\right) \lambda_{q}^{k-(2 i-1)}\left(\lambda_{q}^{2}+4\right)^{2 i} & \text { if } k \text { is odd } .\end{cases}
$$


Proof Let $k$ be even. By (4),

$$
\begin{aligned}
a_{k}= & \frac{1}{\sqrt{\lambda_{q}^{2}+4}}\left[\left(\frac{\lambda_{q}+\sqrt{\lambda_{q}^{2}+4}}{2}\right)^{k}-\left(\frac{\lambda_{q}-\sqrt{\lambda_{q}^{2}+4}}{2}\right)^{k}\right] \\
= & \frac{1}{2^{k-1} \sqrt{\lambda_{q}^{2}+4}}\left[\left(\begin{array}{l}
k \\
1
\end{array}\right) \lambda_{q}^{k-1} \sqrt{\lambda_{q}^{2}+4}+\left(\begin{array}{l}
k \\
3
\end{array}\right) \lambda_{q}^{k-3}\left(\sqrt{\lambda_{q}^{2}+4}\right)^{3}\right. \\
& \left.+\cdots+\left(\begin{array}{c}
k \\
k-1
\end{array}\right) \lambda_{q}\left(\sqrt{\lambda_{q}^{2}+4}\right)^{k-1}\right] \\
= & \frac{1}{2^{k-1}}\left[\left(\begin{array}{c}
k \\
1
\end{array}\right) \lambda_{q}^{k-1}+\left(\begin{array}{l}
k \\
3
\end{array}\right) \lambda_{q}^{k-3}\left(\lambda_{q}^{2}+4\right)^{2}\right. \\
& \left.+\cdots+\left(\begin{array}{c}
k \\
k-1
\end{array}\right) \lambda_{q}\left(\lambda_{q}^{2}+4\right)^{k-2}\right] \\
= & \frac{1}{2^{k-1}}\left[\sum_{i=0}^{\frac{k-2}{2}}\left(\begin{array}{c}
k \\
2 i+1
\end{array}\right) \lambda_{q}^{k-(2 i+1)}\left(\lambda_{q}^{2}+4\right)^{2 i}\right] .
\end{aligned}
$$

Similarly, if $k$ is odd, then we get

$$
a_{k}=\frac{1}{2^{k-1}} \sum_{i=0}^{\frac{k-1}{2}}\left(\begin{array}{c}
k \\
2 i+1
\end{array}\right) \lambda_{q}^{k-(2 i+1)}\left(\lambda_{q}^{2}+4\right)^{i} .
$$

\section{Proposition 11}

$$
{ }_{i=1}^{k+1} a_{i}=\frac{a_{k+2}+a_{k+1}-1}{\lambda_{q}}
$$

and

$$
{ }_{i=1}^{k+1} b_{i}=\frac{b_{k+2}+b_{k+1}-\left(\lambda_{q}+2\right)}{\lambda_{q}} .
$$

Proof From (3), we have

$$
\begin{aligned}
a_{k+2}-a_{k+1} & =\lambda_{q} a_{k+1}+a_{k}-a_{k+1} \\
& =\left(\lambda_{q}-1\right) a_{k+1}+a_{k},
\end{aligned}
$$

and so,

$$
\begin{aligned}
& n=0 \quad \Rightarrow \quad a_{2}-a_{1}=\left(\lambda_{q}-1\right) a_{1}+a_{0}, \\
& n=1 \quad \Rightarrow \quad a_{3}-a_{2}=\left(\lambda_{q}-1\right) a_{2}+a_{1}, \\
& \vdots \\
& n=k-1 \quad \Rightarrow \quad a_{k+1}-a_{k}=\left(\lambda_{q}-1\right) a_{k}+a_{k-1}, \\
& n=k \quad \Rightarrow \quad a_{k+2}-a_{k+1}=\left(\lambda_{q}-1\right) a_{k+1}+a_{k} .
\end{aligned}
$$


If we sum both sides, then we obtain

$$
\begin{aligned}
a_{k+2}-a_{1} & =\left(\lambda_{q}-1\right)\left(a_{1}+a_{2}+\cdots+a_{k+1}\right)+\left(a_{0}+a_{1}+\cdots+a_{k}\right) \\
& =\lambda_{q}\left(a_{1}+a_{2}+\cdots+a_{k+1}\right)+a_{0}-a_{k+1} .
\end{aligned}
$$

Since $a_{0}=0$ and $a_{1}=1$, we have

$$
\begin{aligned}
& a_{k+2}-1=\lambda_{q}\left(a_{1}+a_{2}+\cdots+a_{k+1}\right)-a_{k+1}, \\
& a_{k+2}+a_{k+1}-1=\lambda_{q}\left(a_{1}+a_{2}+\cdots+a_{k+1}\right), \\
& \frac{a_{k+2}+a_{k+1}-1}{\lambda_{q}}=a_{1}+a_{2}+\cdots+a_{k+1}, \\
& { }_{i=1}^{k+1} a_{i}=\frac{a_{k+2}+a_{k+1}-1}{\lambda_{q}} .
\end{aligned}
$$

Similarly, it is easily seen that

$$
{ }_{i=1}^{k+1} b_{i}=\frac{b_{k+2}+b_{k+1}-\left(\lambda_{q}+2\right)}{\lambda_{q}} .
$$

\section{Polynomial representations of $a_{k}$ and $b_{k}$}

Before we find the polynomial representations of $a_{k}$ and $b_{k}$, note the following identities

$$
\left(\begin{array}{l}
k \\
p
\end{array}\right)+2\left(\begin{array}{c}
k+1 \\
p-1
\end{array}\right)-\left(\begin{array}{c}
k \\
p-2
\end{array}\right)=\left(\begin{array}{c}
k+2 \\
p
\end{array}\right)
$$

and

$$
\left(\begin{array}{l}
k \\
p
\end{array}\right)+\left(\begin{array}{l}
k-1 \\
p-1
\end{array}\right)=\left(\begin{array}{l}
k-1 \\
p-1
\end{array}\right) \frac{p+k}{p}
$$

Theorem 1 Let $\left\{a_{k}\right\}$ denote the generalized Fibonacci sequence. Then, the polynomial representations of $a_{2 k}$ and $a_{2 k+1}$ are

$$
\begin{aligned}
a_{2 k}= & \left(\lambda_{q}\right)^{2 k-1}+\left(\begin{array}{c}
2 k-2 \\
1
\end{array}\right)\left(\lambda_{q}\right)^{2 k-3}+\left(\begin{array}{c}
2 k-3 \\
2
\end{array}\right)\left(\lambda_{q}\right)^{2 k-5} \\
& +\cdots+\left(\begin{array}{c}
k+2 \\
k-3
\end{array}\right)\left(\lambda_{q}\right)^{3}+\left(\begin{array}{c}
k+1 \\
k-2
\end{array}\right)\left(\lambda_{q}\right)
\end{aligned}
$$

and

$$
\begin{aligned}
a_{2 k+1}= & \left(\lambda_{q}\right)^{2 k}+(2 k-1)\left(\lambda_{q}\right)^{2 k-2}+\left(\begin{array}{c}
2 k-2 \\
1
\end{array}\right) \frac{2 k-3}{2}\left(\lambda_{q}\right)^{2 k-4} \\
& +\left(\begin{array}{c}
2 k-3 \\
2
\end{array}\right) \frac{2 k-5}{3}\left(\lambda_{q}\right)^{2 k-6}+\cdots+\left(\begin{array}{c}
k+1 \\
k-2
\end{array}\right) \frac{3}{k-1}\left(\lambda_{q}\right)^{2}+1 .
\end{aligned}
$$

Proof We will use the induction method on $k$. For $k=1$, we have $a_{2}=\lambda_{q}$, and for $k=2$, we have $a_{4}=\left(\lambda_{q}\right)^{3}+2 \lambda_{q}$. Now, suppose that the equality is true for $k=1,2, \ldots, n$. We will 
show that it holds for $k=n+1$. By assumption,

$$
\begin{aligned}
a_{2 n-2}= & \left(\lambda_{q}\right)^{2 n-3}+\left(\begin{array}{c}
2 n-4 \\
1
\end{array}\right)\left(\lambda_{q}\right)^{2 n-5}+\left(\begin{array}{c}
2 n-5 \\
2
\end{array}\right)\left(\lambda_{q}\right)^{2 n-7} \\
& +\cdots+\left(\begin{array}{c}
n+1 \\
n-4
\end{array}\right)\left(\lambda_{q}\right)^{3}+\left(\begin{array}{c}
n \\
n-3
\end{array}\right)\left(\lambda_{q}\right)
\end{aligned}
$$

and

$$
\begin{aligned}
a_{2 n}= & \left(\lambda_{q}\right)^{2 n-1}+\left(\begin{array}{c}
2 n-2 \\
1
\end{array}\right)\left(\lambda_{q}\right)^{2 n-3}+\left(\begin{array}{c}
2 n-3 \\
2
\end{array}\right)\left(\lambda_{q}\right)^{2 n-5} \\
& +\cdots+\left(\begin{array}{c}
n+2 \\
n-3
\end{array}\right)\left(\lambda_{q}\right)^{3}+\left(\begin{array}{c}
n+1 \\
n-2
\end{array}\right)\left(\lambda_{q}\right) .
\end{aligned}
$$

From (9), we have $a_{2 k+2}=\left(\lambda_{q}^{2}+2\right) a_{2 k}-a_{2 k-2}$, and by definition of $a_{k}$, we get

$$
\begin{aligned}
a_{2 n+2}= & \left(\lambda_{q}^{2}+2\right)\left[\begin{array}{c}
\left(\lambda_{q}\right)^{2 n-1}+\left(\begin{array}{c}
2 n-2 \\
1
\end{array}\right)\left(\lambda_{q}\right)^{2 n-3}+\left(\begin{array}{c}
2 n-3 \\
2
\end{array}\right)\left(\lambda_{q}\right)^{2 n-5} \\
+\cdots+\left(\begin{array}{c}
n+2 \\
n-3
\end{array}\right)\left(\lambda_{q}\right)^{3}+\left(\begin{array}{c}
n+1 \\
n-2
\end{array}\right)\left(\lambda_{q}\right)
\end{array}\right] \\
& -\left[\begin{array}{c}
\left(\lambda_{q}\right)^{2 n-3}+\left(\begin{array}{c}
2 n-4 \\
1
\end{array}\right)\left(\lambda_{q}\right)^{2 n-5}+\left(\begin{array}{c}
2 n-5 \\
2
\end{array}\right)\left(\lambda_{q}\right)^{2 n-7} \\
+\cdots+\left(\begin{array}{c}
n+1 \\
n-4
\end{array}\right)\left(\lambda_{q}\right)^{3}+\left(\begin{array}{c}
n \\
n-3
\end{array}\right)\left(\lambda_{q}\right)
\end{array}\right] \\
= & \left(\lambda_{q}\right)^{2 n+1}+\left(\left(\begin{array}{c}
2 n-2 \\
1
\end{array}\right)+2\right)\left(\lambda_{q}\right)^{2 n-1}+\left[\left(\begin{array}{c}
2 n-3 \\
2
\end{array}\right)+2\left(\begin{array}{c}
2 n-2 \\
1
\end{array}\right)\right]\left(\lambda_{q}\right)^{2 n-3} \\
& +\cdots+\left[\left(\begin{array}{c}
n+1 \\
n-2
\end{array}\right)+2\left(\begin{array}{c}
n+2 \\
n-3
\end{array}\right)\right]\left(\lambda_{q}\right)^{3}+2\left(\begin{array}{c}
n+1 \\
n-2
\end{array}\right) \lambda_{q} .
\end{aligned}
$$

From (21), we get

$$
\begin{aligned}
a_{2 n+2}= & \left(\lambda_{q}\right)^{2 n+1}+\left(\begin{array}{c}
2 n \\
1
\end{array}\right)\left(\lambda_{q}\right)^{2 n-1}+\left(\begin{array}{c}
2 n-1 \\
2
\end{array}\right)\left(\lambda_{q}\right)^{2 n-3} \\
& +\cdots+\left(\begin{array}{c}
n+3 \\
n-2
\end{array}\right)\left(\lambda_{q}\right)^{3}+\left(\begin{array}{c}
n+2 \\
n-1
\end{array}\right)\left(\lambda_{q}\right) .
\end{aligned}
$$

Now, we compute $a_{2 k+1}$. By definition of $a_{k}$, we get

$$
\begin{aligned}
a_{2 k+1}= & \frac{1}{\lambda_{q}}\left(a_{2 k+2}-a_{2 k}\right) \\
= & \frac{1}{\lambda_{q}}\left[\begin{array}{c}
\left(\left(\lambda_{q}\right)^{2 k+1}+\left(\begin{array}{c}
2 k \\
1
\end{array}\right)\left(\lambda_{q}\right)^{2 k-1}+\left(\begin{array}{c}
2 k-1 \\
2
\end{array}\right)\left(\lambda_{q}\right)^{2 k-3}\right. \\
\left.+\cdots+\left(\begin{array}{c}
k+3 \\
k-2
\end{array}\right)\left(\lambda_{q}\right)^{3}+\left(\begin{array}{c}
k+2 \\
k-1
\end{array}\right)\left(\lambda_{q}\right)\right) \\
-\left(\left(\lambda_{q}\right)^{2 k-1}+\left(\begin{array}{c}
2 k-2 \\
1
\end{array}\right)\left(\lambda_{q}\right)^{2 k-3}+\left(\begin{array}{c}
2 k-3 \\
2
\end{array}\right)\left(\lambda_{q}\right)^{2 k-5}\right. \\
\left.+\cdots+\left(\begin{array}{c}
k+2 \\
k-3
\end{array}\right)\left(\lambda_{q}\right)^{3}+\left(\begin{array}{c}
k+1 \\
k-2
\end{array}\right)\left(\lambda_{q}\right)\right)
\end{array}\right] .
\end{aligned}
$$

From (22), we get

$$
\begin{aligned}
a_{2 k+1}= & \left(\lambda_{q}\right)^{2 k}+(2 k-1)\left(\lambda_{q}\right)^{2 k-2}+\left(\begin{array}{c}
2 k-2 \\
1
\end{array}\right) \frac{2 k-3}{2}\left(\lambda_{q}\right)^{2 k-4} \\
& +\left(\begin{array}{c}
2 k-3 \\
2
\end{array}\right) \frac{2 k-5}{3}\left(\lambda_{q}\right)^{2 k-6}+\cdots+\left(\begin{array}{c}
k+1 \\
k-2
\end{array}\right) \frac{3}{k-1}\left(\lambda_{q}\right)^{2}+1 .
\end{aligned}
$$


Theorem 2 Let $\left\{b_{k}\right\}$ denote the generalized Lucas sequence. Then, the polynomial representations of $b_{2 k}$ and $b_{2 k+1}$ are

$$
\begin{aligned}
b_{2 k}= & \left(\lambda_{q}\right)^{2 k}+(2 k)\left(\lambda_{q}\right)^{2 k-2}+\left(\begin{array}{c}
2 k-3 \\
1
\end{array}\right) \frac{2 k}{2}\left(\lambda_{q}\right)^{2 k-4} \\
& +\left(\begin{array}{c}
2 k-4 \\
2
\end{array}\right) \frac{2 k}{3}\left(\lambda_{q}\right)^{2 k-6}+\cdots+\left(\begin{array}{c}
k \\
k-2
\end{array}\right) \frac{2 k}{k-1}\left(\lambda_{q}\right)^{2}+2
\end{aligned}
$$

and

$$
\begin{aligned}
b_{2 k+1}= & \left(\lambda_{q}\right)^{2 k+1}+(2 k+1)\left(\lambda_{q}\right)^{2 k-1}+\left(\begin{array}{c}
2 k-2 \\
1
\end{array}\right) \frac{2 k+1}{2}\left(\lambda_{q}\right)^{2 k-3} \\
& +\left(\begin{array}{c}
2 k-3 \\
2
\end{array}\right) \frac{2 k+1}{3}\left(\lambda_{q}\right)^{2 k-5}+\cdots+\left(\begin{array}{l}
k+1 \\
k-2
\end{array}\right) \frac{2 k+1}{k-1}\left(\lambda_{q}\right) .
\end{aligned}
$$

Proof From (10), it is easy to find the polynomial representations of $b_{2 k}$ and $b_{2 k+1}$.

\section{Competing interests}

The authors declare that they have no competing interests.

\section{Authors' contributions}

All authors completed the paper together. All authors read and approved the final manuscript.

\section{Author details}

${ }^{1}$ Fen-Edebiyat Fakültesi, Matematik Bölümü, Balıkesir Üniversitesi, Balıkesir, 10145, Turkey. ${ }^{2}$ Köprübasi Meslek Yüksek Okulu, Celal Bayar Üniversitesi, Manisa, 45930, Turkey.

Received: 22 January 2013 Accepted: 30 July 2013 Published: 22 August 2013

\section{References}

1. Hecke, E: Über die bestimmung dirichletscher reichen durch ihre funktionalgleichungen. Math. Ann. 112, 664-699 (1936)

2. Cangül, IN, Singerman, D: Normal subgroups of Hecke groups and regular maps. Math. Proc. Camb. Philos. Soc. 123(1), 59-74 (1998)

3. Lang, ML: Normalizers of the congruence subgroups of the Hecke groups $G_{4}$ and $G_{6}$. J. Number Theory 90(1), 31-43 (2001)

4. Sahin, R, Bizim, O, Cangul, IN: Commutator subgroups of the extended Hecke groups $\bar{H}\left(\lambda_{q}\right)$. Czechoslov. Math. J. 54(129)(1), 253-259 (2004)

5. Sahin, R, Bizim, O: Some subgroups of the extended Hecke groups $\bar{H}\left(\boldsymbol{\lambda}_{q}\right)$. Acta Math. Sci. 23(4), 497-502 (2003)

6. Koruoğlu, Ö, Sahin, R: Generalized Fibonacci sequences related to the extended Hecke groups and an application to the extended modular group. Turk. J. Math. (2009). doi:10.3906/mat-0902-33

7. Djordjević, GB, Srivastava, HM: Some generalizations of the incomplete Fibonacci and the incomplete Lucas polynomials. Adv. Stud. Contemp. Math. 11, 11-32 (2005)

8. Djordjević, GB, Srivastava, HM: Incomplete generalized Jacobsthal and Jacobsthal-Lucas numbers. Math. Comput. Model. 42, 1049-1056 (2005)

9. Djordjević, GB, Srivastava, HM: Some generalizations of certain sequences associated with the Fibonacci numbers. J. Indonesian Math. Soc. 12, 99-112 (2006)

10. Edson, M, Yayenie, O: A new generalization of Fibonacci sequence and extended Binet's formula. Integers 9(6), 639-654 (2009)

11. Mushtaq, Q, Hayat, U: Horadam generalized Fibonacci numbers and the modular group. Indian J. Pure Appl. Math. 38(5), 345-352 (2007)

12. Raina, RK, Srivastava, HM: A class of numbers associated with the Lucasnumbers. Math. Comput. Model. 25(7), 15-22 (1997)

13. Vajda, S: Fibonacci \& Lucas Numbers, and the Golden Section: Theory and Applications. Ellis Horwood, Chichester (1989)

14. Yilmaz Özgür, N: Generalizations of Fibonacci and Lucas sequences. Note Mat. 21(1), 113-125 (2002)

15. Yilmaz Özgür, N: On the sequences related to Fibonacci and Lucas numbers. J. Korean Math. Soc. 42(1), 135-151 (2005) 